\title{
Historisch vergleichen, aber richtig. Irak ist nicht Westdeutschland
}

\author{
Sonja Grimm
}

Zusammenfassung: Die Demokratisierung Westdeutschlands nach dem Zweiten Weltkrieg unter alliierter Besatzung wird häufig als Modell für die irakische Transformation unter US-Besatzung herangezogen. Dies ist methodisch fragwürdig, verkennt den veränderten Kontext und lässt außer Acht, welche günstigen und weniger günstigen Bedingungen Westdeutschland im Jahr 1945 vom Irak im Jahr 2003 unterscheiden. Hätten die US-Planer systematisch verglichen, so hätten sie wichtige Erkenntnisse über Erfolgsbedingungen und Hemmnisse extern beaufsichtigter Demokratisierung gewinnen können. Diese betreffen die strukturellen und kontextuellen Gegebenheiten, unter denen sich die Demokratisierung vollzieht, die Bedeutung eines legitimen Eingreifens in die internen Belange eines Gemeinwesens und das fragile Verhältnis zwischen internen und externen Akteuren während der Besatzung.

Schlïsselwörter: Besatzung · Irak · Westdeutschland · Demokratisierung · Demokratieförderung $\cdot$ Historischer Vergleich

\section{Comparative historical analysis, but done well. Iraq is nothing like West Germany}

\begin{abstract}
The democratization of West Germany after World War II under Allied occupation is often taken as a role model for the Iraqi transition to democracy under US occupation. This method, which is disputable, overlooks the changing international context and negates the favourable and unfavourable conditions that distinguish West Germany in 1945 from Iraq in 2003. If US officials had systematically compared both cases, they would have learned a great deal about con-
\end{abstract}

Ich danke den Mitgliedern des Forschungsnetzwerks „Externe Demokratisierungspolitik“ sowie den Mitgliedern der Abteilung „Demokratie: Strukuren, Leistungsprofil und Herausforderungen“ und der Forschungsgruppe „Zivilgesellschaft, Citizenship und politische Mobilisierung in Europa“ am Wissenschaftszentrum Berlin für Sozialforschung (WZB) für hilfreiche Kommentare zu einer früheren Version dieses Beitrags. Ebenso ein herzliches Dankeschön an die beiden anonymen Gutachter der Zeitschrift für Vergleichende Politikwissenschaft.

Dr. S. Grimm (ه)

Fachbereich Politik- und Verwaltungswissenschaft, Universität Konstanz, Fach 86,

78457 Konstanz, Deutschland

Tel.: +49-7531-883649

Fax: +49-7531-882774

E-Mail: sonja.grimm@uni-konstanz.de 
ditions that contribute to success and about obstacles to external democratization. These include the structural and contextual conditions of a nation's transition to democracy, the importance of a legitimate intervention in the internal affairs of a community, and the fragile relationship between internal and external actors during an occupation.

Keywords: Occupation · Iraq · West Germany · Democratization · Promotion of democracy · Comparative historical analysis

\section{Einleitung}

Die Demokratisierung Westdeutschlands nach dem Zweiten Weltkrieg unter alliierter Besatzung wird unerfreulich häufig in Politik, Medien und Wissenschaft als Erfolgsfall und zugleich als Vorbild für die irakische Transformation nach der US-Intervention im Jahr 2003 genannt. Politiker wie die damalige US-Außenministerin Condoleezza Rice (Benjamin 2003) oder der damalige stellvertretende US-Verteidigungsminister Paul Wolfowitz (Wolfowitz 2004) bemühten den historischen Vergleich, um die Politik der USA im Irak zu rechtfertigen. Der Politikwissenschaftler James Dobbins stützt die Aussage, Demokratie könne erzwungen werden, in seinen lessons learned aus den US-amerikanischen Versuchen der Nationenbildung maßgeblich auf den deutschen Fall (Dobbins et al. 2003, S. 149-166). Ähnlich argumentieren die Wissenschaftler Schoch (2006, S. 18) und Jackson (2004, S. 27-30), und es lassen sich einige Belege dafür finden, dass sich Mitglieder der US-Regierung sowie der Coalition Provisional Authority (CPA) in Bagdad mit dem Studium der Besatzung Westdeutschlands in den Jahren 1945 bis 1949 auf ihre Aufgabe im Irak vorbreitet haben. Nicht ohne Entsetzen beschreibt Noah Feldman, Berater der CPA für Verfassungsfragen, wie er auf seinem Flug nach Bagdad aktuelle Lektüre über die Golfregion konsultierte, während seine Kollegen ausnahmslos Bücher über die amerikanische Besatzung und Rekonstruktion von Deutschland und Japan gelesen hätten (Feldman 2004, S. 1).

Ohne Zweifel sind historische Vorläufer ein gutes Studienobjekt. Es ist allerdings vermessen und voreilig, die Instrumente und Strategien eines vergangenen Erfolges auf einen aktuellen Fall zu übertragen und dabei auf einen ähnlichen Erfolg zu hoffen. Dieses Vorgehen ist methodisch fragwürdig, verkennt die veränderten Kontextbedingungen und lässt außer Acht, welche für die Re- oder Neudemokratisierung günstigen und weniger günstigen Bedingungen Westdeutschland im Jahr 1945 vom Irak im Jahr 2003 unterscheiden. Der folgende Beitrag will hier Aufklärung leisten und fragt daher am Beispiel des Vergleichs Westdeutschland - Irak: Welchen Erkenntnisgewinn bringt der historische Vergleich? Was hätten US-Planer vor der Besatzung des Irak tatsächlich aus dem deutschen Fall lernen können?

Der Beitrag vertritt folgende These: US-Planer hätten in fünf zentralen Bereichen wichtige Erkenntnisse aus dem Studium der US-Besatzung Westdeutschlands ziehen können. Dies betrifft (1) die Bedeutung eines legitimen externen Eingreifens, (2) die Rolle der strukturellen Bedingungen, woraus sich Erblasten und Ressourcen für die Demokratisierung ergeben, (3) die Rolle der externen Kontextbedingungen, die einerseits zu Katalysatoren, andererseits zu Hindernissen für die Demokratisierung werden können, (4) das Verhältnis zwischen den externen und den internen Akteuren während der Besat- 
zung und (5) den Preis, den interne und externe Akteure für die Demokratisierung unter Besatzung bezahlen. Aus dem Vergleich in Abschnitt 2 wird deutlich werden, wie wenig sich Westdeutschland 1945 und der Irak 2003 gleichen und dass damit die Demokratisierungschancen unter Anwendung des westdeutschen Demokratisierungspfads für den Irak eher gering sind. Theoretische Überlegungen zum Nutzen des historischen Vergleichs in Abschnitt 3 runden den Beitrag ab.

\section{Demokratisierung unter Besatzung: Westdeutschland und der Irak im Vergleich}

Höchst unterschiedliche Konfliktlagen führten im Falle Westsdeutschlands und im Falle des Irak zu einer militärischen Invasion und der sich anschließenden Besatzung. Entsprechend unterschiedlich gestaltete sich die Ausgangslage für die externen Akteure zu Beginn ihrer Besatzungszeit. Dies betrifft die Legitimität des externen Eingreifens und der Besatzung (Abschnitt 2.1), die Erblasten und Ressourcen für eine mögliche Demokratisierung (Abschnitt 2.2) und die externen Kontextbedingungen (Abschnitt 2.3). All diese von der Transitionsforschung und der Forschung zu Staatsaufbau als relevant identifizierten Faktoren variieren zwischen den beiden Fällen erheblich. Folgt man der einschlägigen Literatur zur systematischen Auswahl von Fällen für die politikwissenschaftliche Analyse (Przeworksi u. Teune 1970, S. 31-39) und für strukturierte und fokussierte Vergleiche (George u. Bennet 2005, S. 67-72), verbietet sich eine unreflektierte Analogie der beiden Fälle, was die folgende Darstellung veranschaulicht.

\subsection{Legitimität des externen Eingreifens und der Besatzung}

Externe Akteure greifen durch eine militärische Intervention und eine Besatzung tief in die internen Belange des Ziellandes ein. Um Gehör zu finden und Entscheidungen erfolgreich implementieren zu können, bedürfen sie dabei eines „Legitimitätskredits“, das heißt, die Bevölkerung muss die Anwesenheit der Besatzer zumindest akzeptieren und sich temporär deren Entscheidungsgewalt unterwerfen. Sie muss die Besatzer für eine gewisse Zeit als legitime Regierung anerkennen. Andernfalls sind die Besatzer handlungsunfähig, wenn sie ihre Ziele nicht ausschließlich mit Gewalt durchsetzen wollen (Grimm 2010, S. 114-118; Lipset 1962, S. 70-77). Einen hohen Legitimitätskredit erwerben sich Besatzer noch vor Beginn politischer Handlungen durch eine legale und erfolgreiche militärische Intervention, also eine Intervention, die anerkanntem Völkerrecht entspricht und die ihre Ziele effektiv erreicht (Schaller 2006, S. 15-17). Wie stellt sich diese Ausgangsbedingung in den beiden Ländern dar?

Die Invasion der US-Armee in Deutschland stellte den Höhepunkt des alliierten Kampfes gegen das Regime Adolf Hitlers und seiner Nationalsozialistischen Deutschen Arbeiterpartei (NSDAP) dar. Hitler hatte von Beginn seiner Herrschaft im Jahr 1933 an eine expansive national-chauvinistische Politik verfolgt, mit dem Ziel, „Lebensraum im Osten“" zu erobern und die ,jüdisch-bolschewistische Sowjetunion“ zu zerschlagen (Müller 2004, S. 112). Zum 1. September 1939 marschierte die deutsche Wehrmacht in Polen ein, was den Zweiten Weltkrieg auslöste. Nach dem japanischen Angriff auf Pearl Harbour und angesichts der Bedrängnis, in die Großbritannien und Frankreich nach dem 
jahrelangen Krieg gegen die Nazi-Diktatur geraten waren, traten die USA 1941 in den Krieg ein (vgl. Shirer 1981, S. 892-897). Ihre Truppen landeten am 6. Juni 1944 in der Normandie, eroberten sukzessive die von der Wehrmacht besetzten Gebiete zurück und erreichten schließlich durch ihre militärische und strategische Überlegenheit am 8. Mai 1945 die Kapitulation des Deutschen Reiches (ausführlicher vgl. Müller 2004, S. 198201, 307-321; Schreiber 2007, S. 73-75).

Aus dieser Konfliktsituation heraus ergab sich für die US-Besatzer im Jahr 1945 ein hoher Legitimitätskredit. Die Legalität des militärischen Eingreifens der USA in den Zweiten Weltkrieg und die Notwendigkeit einer Besatzung Deutschlands nach der bedingungslosen Kapitulation der deutschen Reichsführung ist nicht zu bezweifeln (vgl. auch Hoffmann 2007, S. 974). Das Vorliegen einer schwerwiegenden Bedrohung durch das aggressiv operierende Nazi-Regime war unübersehbar. Es hatte eine faschistisch-autoritäre Expansionspolitik und die systematische Ermordung der europäischen Juden betrieben und seine Ideologie mit der Besetzung Polens, der Benelux-Staaten und Frankreichs verbreitet. Die USA verfolgten mit ihrer Intervention die Absicht, dieser aggressiven Politik ein Ende zu setzen, die vom Krieg schwer angeschlagenen Verbündeten zu unterstützen und Europa von der Nazi-Diktatur zu befreien. Der Einsatz militärischer Mittel war dafür notwendig, und die Verbrechen des Nazi-Regimes wogen schwer, so dass eine Besatzung Deutschlands zum Zwecke der Entnazifizierung und Demokratisierung rechtmäßig war. Es gelang den westlichen Alliierten mit Hilfe eines immensen Truppenaufgebots unmittelbar mit dem Einmarsch in Deutschland für die öffentliche Sicherheit zu sorgen und das Gewaltmonopol zu übernehmen.

Die US-Streitkräfte verfügten 2003 im Falle des Irak nicht über einen vergleichbaren Legitimitätskredit. Zweifellos regierte Saddam Hussein mit seiner Baath-Partei den Irak mit unvorstellbarer Grausamkeit und errichtete in den Jahren seiner Herrschaft eine „Republik der Angst" (Makiya 1998), gestützt auf ein repressives Terrorregime, hoch privilegierte Sicherheitskräfte und eine erdölbasierte Rentenwirtschaft (Farouk-Sluglett u. Sluglett 2003). Jedoch war die Invasion der USA in den Irak im Jahre 2003 umstritten.

Nach den terroristischen Anschlägen auf das World Trade Center in New York und das Pentagon in Washington, D.C. am 11. September 2001 verkündete die damalige neokonservative Regierung in Washington, dass die Beseitigung des irakischen Diktators und der demokratische Regimewechsel im Irak notwendige Schritte zur Bekämpfung des internationalen Terrorismus darstellten (Kagan u. Kristol 2004; Rice 2003, S. 112). Die von US-Außenminister Colin Powell im UN-Sicherheitsrat im Februar 2003 vorgetragene Begründung, der Irak besitze Massenvernichtungswaffen und bereite deren Einsatz vor und unterhalte außerdem Beziehungen zu terroristischen Vereinigungen, überzeugte die Mehrheit der Mitglieder des UN-Sicherheitsrats nicht (SC/7696 2003).

Verschiedene Kritiker vermuteten unlautere Motive hinter der Intervention: Nicht Frieden und Demokratie seien das Ziel, sondern die Kontrolle über die im Irak vorhandenen, für die USA strategisch wichtigen Erdöl- und Erdgasreserven. Zudem sei die Regierung Bush von der Hybris getrieben, durch die Errichtung einer Modell-Demokratie im Irak eine Demokratisierungswelle im ganzen Mittleren Osten auszulösen und damit der Region Demokratie, Freiheit und Wohlstand zu bringen (Dunn 2003, S. 280-281). Keinen der beiden Vorwürfe konnte die Bush-Regierung in der Folgezeit überzeugend ausräumen. Auch wurde angezweifelt, ob bereits alle vorhandenen Mittel der interna- 
tionalen Diplomatie gegenüber dem Irak ausgeschöpft seien (Tomuschat 2003, S. 45). Das internationale Sanktionsregime hatte ohne Frage verheerende Auswirkungen auf die Lebensumstände der irakischen Bevölkerung (Sponeck 2005, S. 324-336) und zugleich nur partielle Wirkung auf die Kooperationsbereitschaft der Regierung Saddam Husseins (Duelfer 2004, S. 9). Dennoch glaubte Hans Blix, von 1981 bis 1997 Vorsitzender der Internationalen Atomenergiebehörde und von 2002 bis 2003 Chef der UNSCOM-Nachfolgemission UNMOVIC, eine nunmehr größere Kooperationsbereitschaft des Regimes zu erkennen, als er wenige Tage vor Interventionsbeginn vor den UN-Sicherheitsrat trat (SC/7664 2003). Seiner Ansicht nach dramatisierten die USA die Bedrohung durch den Irak. Und schließlich stand zu befürchten, dass eine militärische Intervention die ohnehin dramatische humanitäre Lage weiter verschlechtern würde (Denison 2003, S. 20). Folgerichtig weigerte sich die Mehrheit der UN-Sicherheitsratsmitglieder, den USA ein Mandat zum Einsatz ,aller nötigen Mittel“ auszustellen.

Die dennoch durchgeführte Militärintervention wurde auf diese Weise zu einem völkerrechtswidrigen Akt der Aggression. Beginnend am 20. März 2003 griffen die USA mit Unterstützung von Truppenkontingenten einer „Koalition der Willigen“ den „Schurkenstaat Irak" an (Bush 2002). Das Regime kollabierte binnen Wochen (Jawad 2005, S. 37). Irakische Soldaten desertierten, die irakische Führungsspitze floh aus Bagdad und die Bevölkerung begrüßte teils freudig, teils vorsichtig abwartend die „Befreier“.

Die Mehrheit der Iraker war erleichtert, den ungeliebten Diktator mittels der US-Invasion losgeworden zu sein. Jedoch rechneten die Iraker nicht mit einer Besatzung ihres Landes. Das Ziel, aus dem Irak eine - nach den Worten von US-Präsident Bush - „musterhafte muslimische Demokratie“" zu machen, stieß bei der Mehrheit der Iraker auf wenig Verständnis (Fontan 2006, S. 221-224). Die gut gemeinte Initiierung eines Demokratisierungsprozesses war damit von Beginn an mit dem Makel einer illegitimen militärischen und politischen Intervention behaftet, die zudem von Beginn an ineffektiv durchgeführt wurde. Die US-Truppen waren im Unterschied zur Besatzung Westdeutschlands 1945 unmittelbar nach ihrem Einmarsch in den Irak nicht in der Lage, die öffentliche Sicherheit vollständig zu garantieren und das Gewaltmonopol zu übernehmen (Byman 2004; Dawisha 2004, S. 8-9; Mann 2003, S. 52-56). Über einen sonderlich großen Legitimitätskredit verfügten die US-Truppen zu Beginn der Besatzung also nicht.

\subsection{Strukturelle Bedingungen: Erblasten und Ressourcen für die Demokratisierung}

Die Transitionsforschung hat in den letzten dreißig Jahren vier Faktorenbündel als zentral für eine rasche Institutionalisierung und Konsolidierung von Demokratien identifiziert: erstens hinsichtlich der sozioökonomischen Faktoren ein hoher Grad der Modernisierung und Entwicklung (Lipset 1959; Przeworski u. Limongi 1997; Vanhanen 1983), zweitens hinsichtlich der soziokulturellen Faktoren eine weitgehende ethnische Homogenität, ein hohes Maß an innergesellschaftlichem Vertrauen und das Nichtvorhandensein gravierender Konfliktlinien (Huntington 1991; Putnam 1993), drittens hinsichtlich des institutionellen Erbes das Vorhandensein demokratischer und rechtsstaatlicher Vorerfahrungen (Merkel 1999, S. 199) und viertens hinsichtlich des Grads der Staatlichkeit das Vorhandensein eines funktionierenden Staates und eines Konsenses über die Nationswerdung (Higley u. Burton 1989; Przeworski 1991; S. 51-99; Rotberg 2004). 
Angesichts der besonderen Problemlagen, mit denen eine Nachkriegsgesellschaft konfrontiert ist, fügen Kriegsursachenforscher außerdem eine fünfte Bedingung hinzu, die sich auf den Kriegsausgang bezieht, nämlich dass die Kriegsschuldfrage eindeutig beantwortet werden kann und über Sieg und Niederlage im Krieg Einigkeit besteht (Walter 1997; Hartzel et al. 2001). Sind die genannten Bedingungen nicht erfüllt, so summieren sie sich in den Augen der Transitions- und Kriegsursachenforscher zu beträchtlichen Hemmnissen für eine rasche Demokratisierung (Croissant 2002, S. 53-63; Merkel et al. 2003, S. 200-238).

Für Deutschland lassen sich strukturelle Bedingungen identifizieren, die sich in den Jahren 1945 bis 1949 als Ressourcen für die Demokratisierung unter alliierter Aufsicht erwiesen. Im Unterschied dazu sind im Irak im Jahr 2003 deutlich weniger Ressourcen, aber einige gravierende Erblasten zu erkennen. Für Deutschland erwiesen sich (1) die Erfahrung der Kriegsschuld, (2) die ethnische Homogenität, (3) die Vorerfahrung mit der Weimarer Demokratie sowie ihrem Zusammenbruch und der sich daraus ergebende Grundkonsens über zentrale Streitfragen, (4) der Grad der vorhandenen Staatlichkeit einschließlich einer effektiven Verwaltung und (5) die sozioökonomischen Rahmenbedingungen als günstig. Umgekehrt waren diese Faktoren im Irak im Jahr 2003 eher ungünstig ausgeprägt. Wie der folgende Vergleich zeigen wird, hätten die gegebenen strukturellen Ausgangsbedingungen die Verantwortlichen in der US-Regierung zur Vorsicht gegenüber voreiligen Erfolgshoffnungen mahnen müssen.

(1) Die Kriegsschulderfahrung: Der Schock der totalen Niederlage und die späte Erkenntnis, dass Deutsche die systematische Ermordung von mehreren Millionen Juden betrieben hatten, führte nach $1945 \mathrm{zu}$ einer kollektiven Abwehrreaktion gegen alles, was mit dem alten Nazi-Regime zu tun hatte, einschließlich der Leugnung, selbst Anhänger des Regimes gewesen zu sein. Der Wunsch, zu vergessen und über die eigene Beteiligung lieber zu schweigen, bildete das Fundament für die Erneuerung des politischen Systems (Benz 1999b). Das Angebot der Demokratisierung und der Westintegration auf politischer Ebene war daher willkommen. Dies bedeutete jedoch nicht, dass die damit verbundene kulturelle Öffnung hin zum US-amerikanischen Gesellschaftsmodell komplett vollzogen wurde. Vielmehr erlaubte die Mischung aus Kontrolle und Freiheit eine selektive Übernahme passender Wertvorstellungen (Piel 1996, S. 146-150). Eine der deutschen Schuld ähnliche Erfahrung hat das irakische Volk vor der US-Intervention nicht gemacht. Vielmehr sah sich die Mehrheit eher als Opfer des Hussein-Regimes und erhoffte sich eine rasche Stabilisierung der sozioökonomischen Lage. Eine Fremdherrschaft erwartete sie hingegen nicht (Fontan 2006, S. 221-224; Grimm 2010, S. 289).

(2) Zustand der politischen Gemeinschaft: Eine Fragmentierung der politischen Gemeinschaft, wie sie im Irak angesichts lange unterdrückter und nach 2003 eskalierender ethnischer, religiöser und ideologischer Konflikte zu beobachten war, kann so für Westdeutschland im Jahr 1945 nicht festgestellt werden. Die innere wie äußere Nationsbildung war bereits mit der Formierung des Kaiserreichs und der Bismarck'schen Reichseinigungspolitik vor Ausbruch des Ersten Weltkrieges abgeschlossen (Ullmann 1995, S. 14-22). Umstrittene Gebiete an den Grenzen des Deutschen Reiches von 1933, vor allem die ostpreußischen Provinzen und Elsass-Lothringen (Ullmann 1995, S. 26-28), wurden auf den alliierten Konferenzen explizit nicht mehr deutschem Gebiet zugerechnet. Die Bevölkerung in den verbliebenen Gebieten einte eine gemeinsame Sprache und 
eine gemeinsame Geschichte. Entsprechend war damit die Kongruenz von Staatsgebiet, Staatsvolk und Staatsnation im Jahr 1945 gegeben.

Eine vergleichbare Kongruenz von Staatsgebiet, Staatsvolk und Staatsnation gab es im Irak im Jahre 2003 nicht. Sowohl unter den haschemitischen Königen wie auch später unter dem Regime der Baath-Partei blieb die Gesellschaft des Irak fragmentiert, ethnisch zwischen Arabern und Kurden, religiös zwischen Schiiten und Sunniten (Woods 2006, S. 2-3) und wirtschaftlich zwischen den vom Regime Privilegierten (zumeist Sunniten) gegenüber den vom Regime Marginalisierten (zumeist Schiiten und Kurden) sowie zwischen jenen mit Zugang zu den reichhaltigen Erdölvorkommen um Kirkuk im Norden und Basra im Süden und jenen ohne Zugang (Farouk-Sluglett u. Sluglett 2003, S. 270).

Saddam Hussein beteiligte eine breite Mittelschicht an den Renten der Erdölwirtschaft (Stansfield 2007, S. 97). Jenseits dessen marginalisierte er Kurden, assyrische Christen, Turkmenen und schiitische Oppositionelle (Davis 2005b, S. 272). Der Erste Golfkrieg gegen den Iran beförderte die repressive Seite des Regimes aus willkürlicher Gewalt, Folter und massiven Menschenrechtsverletzungen. In Husseins „Republik der

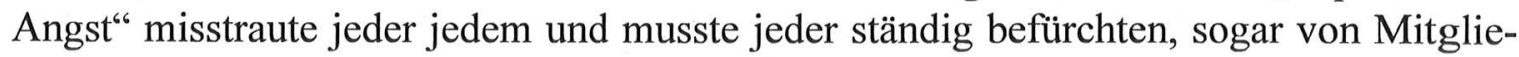
dern der eigenen Familie bei den Geheimdiensten angezeigt zu werden (Woods 2006, S. 3). Hussein schreckte auch nicht davor zurück, rivalisierende Stämme gegeneinander auszuspielen und temporäre Koalitionen mit seinen Feinden einzugehen, wenn es ihm opportun erschien (Baram 1997, S. 5-7, 15). Angesichts dessen standen die Chancen auf eine rasche Demokratisierung nach dem Sturz des Regimes im Jahr 2003 eher schlecht. Die Uneinigkeit der Oppositionsparteien blieb ebenso wie die zentralen Konflikte - beispielsweise der schwelende Streit um die kurdische Selbstbestimmung im Norden und um den Zugang zu Erdöl für die benachteiligten Schiiten - auch nach dem Regimekollaps virulent.

(3) Demokratische Vorerfahrung und demokratischer Grundkonsens: Prägende Erfahrungen im politischen Leben Deutschlands stellten der Niedergang der demokratischen Weimarer Republik und der Aufstieg des faschistischen Hitler-Regimes in den frühen 1930er Jahren dar (Bracher 1955). Dieser Umbruch lag zum Ende des Zweiten Weltkrieges gerade zwölf Jahre zurück. Die Erfahrungen mit dem hochgradig fragmentierten und von Partikularinteressen dominierten Weimarer Parteiensystem und die faschistisch-autoritäre Machtentfaltung des Einparteiensystems erforderten einen radikalen Neubeginn (Lepsius 1978, S. 41-42, 63; Wehler 2003, S. 608). Nicht diskreditierte Eliten waren über alle politischen Strömungen hinweg bereit, eine Systemalternative mit einem gemäßigten demokratischen Parteienwettbewerb zu unterstützen. Sie vereinte eine Art „Anti-Konsens“. Das neue System sollte ,,anti-totalitär“, „,anti-charismatisch“, ,anti-zentralistisch“ und „anti-militaristisch“ sein, auf christlichen und liberalen Werten beruhen und gegen demokratiefeindliche Mobilisierung immun sein. Unter dem Motto „Nie wieder!“ wollten diejenigen, die den Niedergang Weimars noch selbst erlebt hatten, die in der Weimarer Verfassung eingebauten institutionellen Blockaden in einer neuen demokratischen Verfassung vermeiden (Benz 1999a; Thränhardt 1996, S. 32).

Die Geschichte des Irak im 20. Jahrhundert bietet demgegenüber wenige rechtsstaatliche und demokratische Ressourcen, auf die eine politische Neuordnung unter demokratischen Vorzeichen hätte rekurrieren können. Lediglich die Zeit der parlamentarischen Monarchie unter dem haschemitischen Königtum von 1921 bis 1958 kann mit einiger 
Vorsicht als Versuch einer Liberalisierung des politischen Systems angesehen werden, die allerdings nur geringe Auswirkungen auf die Bevölkerung hatte. 1945 versprach das Regime Maßnahmen zur Verbesserung der sozialen Sicherheit und gegen Arbeitslosigkeit, eine Lockerung der Pressezensur und eine Veränderung des Wahlrechts zur besseren Repräsentation der ländlichen Regionen (Tripp 2005, S. 114). Alle Bemühungen um Reform und Liberalisierung wurden jedoch wieder zunichte gemacht (Marr 2004, S. 64). Die Wirren um die Vorherrschaft im Irak zwischen 1958 und 1968 und die 35jährige Baath-Herrschaft nach 1968 zerstörte jegliche Form demokratischer Beteiligung. Die Mitgliedschaft in einer der Oppositionsparteien, unter anderem der beiden kurdischen Parteien PUK und KDP sowie der schiitischen Daawa-Partei und des schiitischen SCIRI, wurde bei Todesstrafe verboten (Jawad 2005). Ein demokratischer Grundkonsens über die Grenzen der Ethnien und Glaubensgemeinschaften hinweg bestand 2003 nicht, ebenso wenig eine gemeinsam Vision über die politische Zukunft des Landes (ICG 2003, S. 8-9).

(4) Staatlichkeit und Verwaltungskapazität: Deutschland konnte im Jahr 1945 auf eine lange Rechtsstaatstradition zurückblicken. Aufgrund vielfältiger Erfahrungen mit lokaler Selbstverwaltung existierten effiziente Verwaltungsstrukturen (Eschenburg 1974). Sie wurden von einer privilegierten Funktionselite getragen, die hochgradig professionalisiert und oftmals mit militärischer Vorbildung ans Werk ging (Eschenburg 1974, S. 72). Viele staatliche Verwaltungsbeamte der mittleren und unteren Ebenen wurden nach dem Krieg rasch wieder in ihr Amt eingesetzt und führten auf effiziente Weise wichtige Aufgaben des Wiederaufbaus aus. Briten und Amerikaner machten davon besonders ausgiebig Gebrauch (Ebsworth 1960, S. 82-93; Krieger 1968, 1979, S. 35). Die hierarchischen Strukturen der Verwaltung, die ungeachtet des Nazi-Terrors gut funktionierten, erwiesen sich im Verlauf der Transformation als stabilisierend und damit unterstützend für die Demokratisierung. Die Integrität des Gesamtstaates stand im Unterschied zum Irak nicht zur Disposition.

Im Irak brachen zwischen und innerhalb der kurdischen, sunnitischen und schiitischen Gemeinschaften nach dem Sturz des Diktators heftige Konflikte um die Einheit des Staates, um Machtansprüche im politischen System, um die religiöse Deutungshoheit und um den Zugang zu den reichhaltigen Erdölvorkommen im Norden und im Süden des Landes aus. Das abrupte Ende der Diktatur und der damit verbundene Zerfall staatlicher Autorität weckten vor allem bei den unterdrückten Schiiten und Kurden große Hoffnung auf Freiheit und eine Verbesserung der Lebensumstände. Sunnitische Eliten fürchteten hingegen um den Verlust ihrer Privilegien. Während seiner Herrschaft hatte Saddam Hussein eine Führerdiktatur etabliert, deren gesamtes Herrschaftssystem auf seine Person und eine kleine sunnitische Führungselite hin ausgerichtet wurde (Farouk-Sluglett u. Sluglett 2003, S. 178-187). Hussein übte exekutive und legislative Macht über Magistrate und Richter aus, die ihm allein rechenschaftspflichtig waren, und Streitkräfte, Polizei, Geheimdienste sowie Baath-Partei-Agenten standen ihm rund um die Uhr zu Diensten. Die Führungsstruktur der Baath-Partei war mit dem Staat identisch (ECOSOC 1999: para. 83). Hussein beschäftigte bereits 1980 rund 20\% der Arbeiterschaft auf Staatskosten, vorrangig im staatlichen Sicherheitsapparat (Makiya 1998, S. 39). Bis 1990 waren rund 40\% der irakischen Haushaltè vom Staatsdienst abhängig (Dodge 2003, S. 68-69). Sie standen nach dem Regimekollaps 2003 über Nacht ohne Einkommen dar. 
Der straff organisierte Militär-, Polizei- und Geheimdienstapparat sicherte repressiv die Herrschaft nach innen. Vor allem die Kurden im Norden des Landes bekamen dies im Laufe der 1980er Jahre angesichts mehrerer Giftgasangriffe auf kurdische Dörfer zu spüren. Nach erneuten Giftgasangriffen gewährte der UN-Sicherheitsrat den Kurden humanitäre Hilfe (S/RES/688 1990) und US-amerikanische und britische Luftstreitkräfte sorgten für die Einhaltung einer unilateral eingerichteten Flugverbotszone nördlich des 36. Breitengrades (Cockayne u. Malone 2006, S. 127). ${ }^{1}$ Dies begünstigte die Entwicklung einer autonom durch die Kurden verwalteten Zone (Davis 2005b, S. 230). Damit existierten im Jahr 2003 de facto ein Kurdenstaat im Norden und die Reste eines von sunnitischen Eliten dominierten repressiven Regimes in den übrigen Landesteilen.

(5) Sozioökonomische Rahmenbedingungen: Ökonomisch betrachtet endete der Zweite Weltkrieg für Deutschland mit zerbombten Städten, Flüchtlingsströmen, einer vollständig auf die Kriegsproduktion ausgerichteten Wirtschaftsstruktur und der Notwendigkeit, die große Zahl der Kriegsrückkehrer wieder in den Erwerbsalltag einzugliedern. Der Krieg hatte große Teile des volkswirtschaftlichen Vermögens vernichtet. Allerdings war es den Nationalsozialisten dem Wirtschaftshistoriker Werner Abelshauser zufolge vor Ausbruch des Krieges gelungen, die deutsche Wirtschaft so umzustrukturieren, dass sie in der Lage war, die für die Kriegsführung nötigen Güter zu produzieren und bis in die ersten Kriegsjahre hinein den allgemeinen Lebensstandard zu heben (Abelshauser 1999, S. 537). Die nationalsozialistische Wirtschaftspolitik hatte dabei mindestens drei nicht intendierte Folgen, die sich später begünstigend auf die Entwicklung Westdeutschlands auswirkten: Erstens erforderte die Umstellung von einer Friedens- auf eine Kriegswirtschaft die Errichtung neuer Industrieanlagen, auf die nach dem Zweiten Weltkrieg aufgebaut werden konnte (Abelshauser 1983, S. 20; 1999, S. 524). Zweitens machte der wirtschaftliche Umbau zur Kriegsvorbereitung die umfassende Aus- und Weiterbildung von Facharbeitern notwendig. Auf die Kenntnisse der qualifizierten Arbeiter konnte nach dem Krieg zurückgegriffen werden (Abelshauser 1999, S. 534), ebenso auf die Arbeitskraft der Kriegsflüchtlinge (Wallich 1979, S. 66). Drittens schuf die Restrukturierung und Zentralisierung der industriellen Produktion eine neue Managergeneration, die einen eigenen, selbstverantwortlichen Stil der Unternehmensführung entwickelte. Während viele Top-Funktionäre im Zuge der Denazifizierung ihre Spitzenfunktionen verloren, trug die „zweite Reihe“ erheblich zum raschen Wiederaufbau der Wirtschaft nach 1945 bei (Abelshauser 1999, S. 532). Auf diese Weise stellte die deutsche Kriegswirtschaft ein wichtiges Reservoir an technischer Ausrüstung und Humankapital dar. Zudem waren die wirtschaftlichen Produktionsstätten trotz massiver alliierter Luftangriffe weit weniger zerstört als die alliierten Besatzer zunächst angenommen hatten (Wallich 1955, S. 6).

Die wirtschaftliche Situation des Irak im Jahr 2003 war durch die Folgen der ersten beiden Golfkriege (Iran-Irak 1980-1988 und Irak-Kuwait 1990-1991) und der im Anschluss an den Zweiten Golfkrieg gegen den Irak verhängten Sanktionen sowie durch

1 Die Flugverbotszone nördlich des 36. Breitengrades wurde ohne ausdrückliche Resolution des UN-Sicherheitsrates von den USA und Großbritannien, aber unter Berufung auf Resolution S/RES/688 (1991), eingerichtet. Hinzu trat zum Schutz der schiitischen Bevölkerung im August 1992 eine weitere Flugverbotszone südlich des 32. Breitengrades. 
die Schwächen einer auf die Ausbeutung von Erdölreserven ausgerichteten Rentenökonomie geprägt.

Die seit 1979 betriebene militärische Aufrüstung und die Kosten des Ersten Golfkrieges setzten das Regime in den späten 1980er Jahren ökonomisch unter Druck (Farouk-Sluglett u. Sluglett 2003, S. 277). Die irakische Invasion in Kuwait 1990 sollte die kuwaitische Erdölproduktion unter Kontrolle Saddam Husseins bringen und auf diese Weise zur Lösung der ökonomischen Probleme beitragen. Genau das Gegenteil war jedoch die Folge, denn die durch den UN-Sicherheitsrat autorisierte „Operation Desert. Storm“ gegen den Irak vernichtete bei Luftangriffen große Teile der irakischen Industrieanlagen und der Infrastruktur. Nach Expertenmeinung wurde die irakische Wirtschaft dadurch auf den Stand der frühen 1960er Jahre zurückgeworfen (Davis 2005b, S. 227).

Die ohnehin schwierige sozioökonomische Lage der Zivilbevölkerung nach den beiden Golfkriegen verschärfte sich angesichts eines strikten internationalen Sanktionsregimes (Mack u. Khan 2000, S. 282; Sponeck 2005). Der Verdacht, der Irak verfüge über chemische, biologische und atomare Massenvernichtungswaffen, und die Weigerung der irakischen Regierung, Waffenkontrolleure in die entsprechenden Anlagen zu lassen, wurden mit dem Einfrieren irakischen Vermögens im Ausland und einem strikten Import- und Export-Embargo beantwortet (S/RES/661 1990). In der Folge mangelte es der Zivilbevölkerung an allem, an Lebensmitteln, Elektrizität, Bildung und Gesundheitsfürsorge. Dies betraf alle Teile der Bevölkerung, Sunniten, Schiiten und Kurden, gleichermaßen (FaroukSluglett u. Sluglett 2003, S. 300; Rowat 2001). Laut UN-Angaben lebten 1995 20\% der Bevölkerung, rund vier Millionen Menschen, in extremer Armut (UNDHA 1995). Auch das 1995 aufgelegte „Oil-for-Food“-Programm konnte daran nichts ändern. Die reichen Erdöl- und Erdgasvorkommen stellten 2003 die größte Ressource für den Wiederaufbau des Irak dar, vorausgesetzt allerdings, dass sich die im Irak lebenden Volksgruppen unter der Besatzung auf die Verteilung der erwirtschafteten Einnahmen einigen würden können. Dies wurde jedoch bis 2008 nicht erreicht.

\subsection{Externe Kontextbedingungen}

Jenseits der in Abschnitt 2.2 genannten Faktoren verweisen jüngere Arbeiten in der Transitionsforschung auch auf die Bedeutung des externen Kontextes für die Demokratisierung (Cederman et al. 2008, S. 516-520; Levitsky u. Way 2006; Whitehead 2004). Externe Kontextbedingungen wurden im westdeutschen Fall zu Katalysatoren des Demokratisierungsprozesses. Im Falle des Irak entwickelten sie sich eher zu zusätzlichen Hindernissen auf dem Weg der Demokratisierung.

Über die Ziele ihrer Politik für Kontinentaleuropa nach dem Zweiten Weltkrieg hatten sich die Alliierten bereits früh geeinigt (Guelke 2003, S. 55). Auf der Jalta-Konferenz vom Februar 1945 sprachen sich die Vereinigten Staaten, Großbritannien und die Sowjetunion für ein freies, demokratisches und selbstbestimmtes Europa aus (Yalta Conference 1945, Section II). Davon wurde Deutschland nicht ausgenommen. In der Direktive des American Joint Chief of Staff vom 28. April 1945 wurde entsprechend die Entnazifizierung und im Gegenzug die Demokratisierung Deutschlands als ein Ziel der Besatzung festgehalten (Dorn 1958, S. 62). Allerdings wurde in der gleichen Direktive auch unmissverständlich klargestellt, dass Deutschland nicht zum „Zwecke seiner Befreiung“ (Benz 
1999b, S. 23), sondern als besiegter Feindesstaat besetzt werden würde. Zunächst war die Skepsis gegenüber der Demokratisierungsfähigkeit der Deutschen groß, vor allem, weil die Alliierten eine fest verankerte anti-demokratische Grundhaltung zu erkennen glaubten (Krieger 1968, S. 35-40; Merkl 1968, S. 77). Der weitere Verlauf der Besatzung zwang die westlichen Alliierten dazu, diese Skepsis gegenüber den Deutschen abzulegen. Immer deutlicher wurde, dass die Zusammenarbeit mit der Sowjetunion keineswegs so reibungslos verlaufen würde wie zunächst erhofft. Der sich abzeichnende Systemkonflikt zwischen West und Ost prägte die Deutschlandpolitik der Westalliierten maßgeblich und trug zu einer beschleunigten Demokratisierung Westdeutschlands bei (vgl. Benz 1999b, S. 24-32).

Im Falle des Irak versuchten die US-Besatzer, das Land innerhalb kürzester Zeit in eine „modellhafte muslimische Demokratie“ zu verwandeln. Neben dem Wertekonflikt um das importierte Konzept der „Demokratie“ trat daher auch eine Auseinandersetzung mit den arabischen Nachbarn, die argwöhnisch beobachteten, wie sich die USA nach 2003 in der Region installierten und die Geschicke des Irak zu bestimmen versuchten. In keinem Falle wollten sie eine weitere Dominanz der USA in der Region dulden und sich die Liberalisierung ihrer Regime vorschreiben lassen (Massarrat 2005, S. 35). Besonders beliebt waren die USA Meinungsumfragen zufolge in der Region bereits im Sommer 2003 nicht (Kirchner 2003, S. 61). Hinzu traten schwelende Konflikte, die das Verhältnis des Irak zu seinen Nachbarn bereits vor der US-Intervention belastet hatten. Die Beziehungen zum Iran waren noch aus den Zeiten des Ersten Golfkriegs belastet. Die Unterstützung der schiitischen Mehrheit im Irak durch die schiitisch dominierte Führung des Iran wurde schon von Saddam Hussein bekämpft. Auch nach der US-Intervention musste die neue irakische Regierung die Einmischung des Iran über die schiitischen Kräfte im Irak befürchten (Zuhur 2006, S. 24-31). Die Türkei an der Nordgrenze des Irak sowie Syrien im Nordwesten und der Iran im Nordosten bemühten sich darum, das Entstehen eines eigenen kurdischen Staates, der durch den Zugang zu Erdöl wirtschaftlich erstarken könnte, zu verhindern (Kramer 2007, S. 52). Und schließlich trübte sich das stets freundschaftliche Verhältnis zu Jordanien ein, da das Land in großer Zahl irakische Flüchtlinge aufgenommen hatte, die sozialen und wirtschaftlichen Folgen jedoch kaum bewältigen konnte (Blanchard et al. 2008, S. 26-27). Aus diesem Umfeld konnten nach 2003 kaum Ressourcen generiert werden, welche die Demokratisierung von außen unterstützten.

Jenseits der in den Abschnitten 2.1, 2.2 und 2.3 beschriebenen Ausgangslage entwickelten sich die beiden Fälle auch während der Besatzungszeit höchst unterschiedlich. Dies wird im folgenden Abschnitt beschrieben.

\subsection{Das Verhältnis zwischen den externen und internen Akteuren}

Drei zentrale Aspekte charakterisieren das Verhältnis zwischen internen und externen Akteuren und beeinflussen die Chancen, dass externe Angebote zur Demokratisierung des Landes auf fruchtbaren lokalen Boden fallen (vgl. Grimm 2010). Es handelt sich (1) um die Glaubwürdigkeit des externen Engagements während der Besatzung und den Grad der Einbindung lokaler Akteure in den Wiederaufbau, (2) um den Umgang mit den alten Eliten und (3) um die wirtschaftlichen Aufbauhilfen. Nur wenn externe Akteure glaubwürdig agieren und lokale Akteure früh und wahrhaftig einbinden, großzügig mit 
den alten Eliten verfahren und sinnvolle wirtschaftliche Aufbauhilfe leisten, befördern sie die Demokratisierung.

(1) Glaubwürdigkeit der externen Akteure und Einbindung der lokalen Akteure: Der Konflikt mit der Sowjetunion zwang die westlichen Alliierten nach dem Zweiten Weltkrieg dazu, ihre Skepsis gegenüber den Deutschen abzulegen und gemeinsam mit ihnen eine Systemalternative gegenüber der sowjetischen Besatzungszone zu etablieren. Daher setzte sich bei den westlichen Alliierten bald ein von liberalen Prinzipien abgeleitetes Besatzungskonzept durch, demgemäß es - soweit wie möglich - Angelegenheit der Deutschen bleiben sollte, über die politische und soziale Struktur des Landes zu bestimmen (Krieger 1979, S. 32). Dadurch gelang es den Westalliierten im Verlauf der Besatzungszeit, die deutschen Eliten zur Annahme alliierter Ratschläge zu bewegen (Merkl 1968, S. 62). Dies schloss allerdings nicht aus, dass die Deutschen in einigen Fragen auch eigene Wege gingen. Bei aller alliierten Kontrolle tolerierte der vor allem von US-Amerikanern und Briten getragene Geist der Freiheit und des Diskurses eine deutsche Interpretation der vorgeschlagenen Reformen (Merk1 1968, S. 90).

Ziel der Westalliierten war es, den politischen Parteienwettbewerb langsam entstehen zu lassen und das erneute Erstarken rechtsextremer Parteien im Keim zu ersticken (Ebsworth 1960, S. 30-34). Der Aufbau demokratischer Institutionen begann daher auf der kommunalen Ebene, und die Zulassung von Parteien wurde strikt lizenziert. Für Bürgermeisterämter und kommunale Verwaltungsposten konnten sich die Deutschen selbst melden und wurden dann von der Militärverwaltung ernannt. Erste Wahlen auf lokaler Ebene fanden in Städten und Gemeinden im Verlauf des Jahres 1946 statt (Thränhardt 1996, S. 53). Bis 1947 waren in allen Ländern die Landtage gewählt, die Wahl zum Bundestag fand 1949 nach der Verabschiedung des Grundgesetzes statt.

Die Formulierung der neuen Verfassung geschah weitgehend in alleiniger Verantwortung der Deutschen. Mit der Übergabe der Frankfurter Dokumente trugen die drei alliierten Militärgouverneure den elf Ministerpräsidenten der Westzonen zum 1. Juli 1948 die Verfassunggebung auf (Frankfurter Dokumente 1948). Die letzte Version des als Provisorium gedachten „Grundgesetzes“ wurde vom Parlamentarischen Rat schließlich am 8. Mai 1949 verabschiedet und vier Tage später von den alliierten Militärgouverneuren akzeptiert. Diese verzichteten darauf, sich über die in den Frankfurter Dokumenten festgehaltenen Grundprinzipien und die begleitende Beobachtung der Verfassungsberatungen hinaus direkt in die Verfassunggebung einzumischen. Ein offizielles Veto gegen bestimmte Abschnitte legten sie nicht ein. Aufgrund der durch die Lizenzierungspolitik geschickt vorbereiteten Auswahl der Führungspersönlichkeiten, des zwischen den Westalliierten und den deutschen Eliten vorhandenen demokratischen Grundkonsenses und der im Parlamentarischen Rat ausgehandelten Kompromisse, die letztlich vor allem den Interessen der USA entsprachen, war dies auch nicht nötig (Fait 2001, S. 109; Gimbel 1968, S. 294-299). Dies alles trug erheblich zur Glaubwürdigkeit der Westalliierten in ihrer Deutschlandpolitik bei und zerstreute den Eindruck von „Siegerjustiz“"

Im Fall des Irak hatte die Glaubwürdigkeit der Besatzer bereits durch die oben beschriebenen illegitime Intervention gelitten. Dieser Eindruck wurde in den ersten Wochen der Besatzung durch das Scheitern der Bemühungen von Jay M. Garner und seines Office for Reconstruction and Humanitarian Assistance (ORHA) sowie durch Fehlentscheidungen des nachfolgenden Leiters der Coalition Provisional Authority (CPA), Paul L. Bremer, 
verstärkt. Unter anderem sagte Bremer im Juni 2003 die lokalen Wahlen ab, mit denen Bürgermeisterposten und Gemeinderäte besetzt werden sollten. Stattdessen wurden von der CPA handverlesene Personen ohne Beratủng mit lokalen Ältesten zu Bürgermeistern ernannt. Dieses undemokratische Vorgehen verschärfte den Unmut über die Besatzung, rief Proteste hervor und trieb den Aufständischen Kampfeswillige zu (Caan et al. 2005, S. 8).

In Reaktion darauf versuchte Bremer, lokale Akteure an den Regierungsgeschäften der CPA zu beteiligten. Zunächst schuf Bremer mittels der Bildung des Interim Governing Council (IGC) ein Gremium, das alle Volksgruppen des Irak repräsentierte (Allawi 2007, S. 166). Der IGC ernannte zum 1. September 200325 Übergangsminister, die gemeinsam das erste irakische Kabinett, den Governing Council (GC), bildeten. Der GC wirkte an der Erstellung des Haushalts mit und erließ am 8. März 2004 mit dem Transitional Administrative Law (TAL) eine Übergangsverfassung, die bis zur Verabschiedung der neuen Verfassung Gültigkeit haben sollte und die den Zeitplan für die Institutionalisierung der Demokratie festlegte (Allawi 2007, S. 166). Der Rat setzte damit den Demokratisierungsprozess in Gang, allerdings auf ausdrücklichen Wunsch und nach den Vorstellungen der USA (Allawi 2007, S. 193). Es war offensichtlich, dass die CPA trotz des Zugeständnisses, lokale Akteure an der Verwaltung des Irak auf nationaler Ebene zu beteiligen, die Zügel in der Hand behielt und all ihren Einfluss geltend machte, um den Irak zu demokratisieren.

Diese Regelungshoheit übte Bremer für die CPA ohne Zögern aus, was sich besonders deutlich bei der Abfassung und der Unterzeichung des TAL offenbarte. Der Text selbst kam weitgehend ohne irakische Mitarbeit oder öffentliche Konsultationen zustande (Papagianni 2007, S. 260). Vielmehr war der Text durchdrungen von US-Vorstellungen über Staatlichkeit, Demokratie und Menschenrechte, weniger von arabischen Traditionen und Werten. Zudem hatte Bremer mit den Kurden separate Verhandlungen geführt, deren Ergebnisse in den Text einflossen (Allawi 2007, S. 221-223). Schiiten und Sunniten kritisierten das intransparente Verfahren und die Bevormundung durch die CPA (Papagianni 2007, S. 262). Schließlich mussten sie sich trotz berechtigter Kritik dem Druck der CPA beugen und das Gesetz gegen ihren Willen unterzeichnen (Diamond 2005, S. 44).

Darüber hinaus führte die CPA eine neue Währung ein, errichtete ein zentrales Bankensystem und bemühte sich um den Aufbau von Schulen, Krankenhäusern, Straßen und einer Kommunikationsinfrastruktur. Fortschritte wurden erkennbar, allerdings wurden in vielen Projekten keine Iraker beschäftigt. Sie blieben arbeitslos, während internationale Firmen lukrative Aufträge erhielten (Allawi 2007, S. 253; Caan et al. 2005, S. 10; SIGIR 2006, S. 94). Dies beförderte wiederum den Unmut über die Besatzungspolitik und bot den Aufständischen neuen Anlass zum Widerstand. Meinungsumfragen zeigten, dass die irakische Bevölkerung das Fehlen erkennbarer Verbesserungen der Lebensumstände, die täglich wahrnehmbare Korruption und die hohe Arbeitslosigkeit der CPA zur Last legten (Crocker et al. 2004).

(2) Umgang mit den alten Eliten: Im deutschen Falle versuchten sich die Westalliierten zunächst an einer umfangreichen Denazifizierung des politischen Systems. Mit einer ihrer ersten Entscheidungen lösten die Besatzer die NSDAP, die SA, die SS und alle sonstigen NS-Organisationen auf. Danach lancierten die alliierten Besatzer in ihren jeweiligen Besatzungszonen ein umfassendes Denazifizierungsprogramm (Bungenstab 
1970; Fürstenau 1969; Herz 1982). Allerdings erwies es sich nur bei den führenden politischen Eliten als effektiv (Elster 2004, S. 54). Die in der Ministerialbürokratie und in der Wirtschaft durchgeführten Denazifizierungsaktionen entwickelten sich dagegen eher zu einer „machine for political rehabilitation“ (Frei 2002, S. 28). Nur die Spitzenfunktionäre wurden durch jüngere Kräfte ersetzt. Auf den mittleren und unteren Ebenen kehrten die meisten Funktionäre und Manager nach einer Phase der Suspendierung bis 1948 auf ihre Plätze zurück, einerseits, weil qualifiziertes Personal dringend benötigt wurde (Hoffmann-Lange 1998, S. 175; Zapf 1966, S. 146), und andererseits, weil die mit der Denazifizierung beschäftigten Behörden heillos überfordert waren (Colvin 1947; Ebsworth 1960, S. 6-17). Durch die rasche Wiedereinsetzung der Beamten, Angestellten und Manager erzielten die Besatzungsbehörden damit in Westdeutschland jene Stabilität, die einem raschen Aufbau sehr zuträglich war (Ebsworth 1960, S. 13; Montgomery 1957, S. 184-185). Sie nahmen dafür in Kauf, ehemalige Parteimitglieder und Anhänger des alten Regimes in den Staatsdienst zurückkehren zu lassen (Eschenburg 1974; Prowe 1993, S. 308).

Im Irak wurde mit der „Debaathifizierung“ hingegen der gegenteilige Effekt, nämlich eine Destabilisierung der Lage, erreicht. Mit der Auflösung der Baath-Partei am 16. Mai 2003 (CPA/2003/Oder 1) und der Auflösung der „Entitäten“ im Sicherheitsbereich sowie der Verwaltung am 23. Mai 2003 (CPA/2003/Order 2) wurde der Prozess der Debaathifizierung in Gang gesetzt. Amtsinhaber und Entscheidungsträger der Baath-Partei auf den vier höchsten Stufen der parteiinternen Hierarchie wurden aus ihren Ämtern entfernt und mit einem Beschäftigungsverbot im öffentlichen Sektor belegt. Alle Mitglieder der irakischen Ministerien, des Militärs und der Sicherheitskräfte, der Republikanischen Garden sowie der Geheimdienste wurden freigesetzt und standen danach ohne Aussicht auf Neuanstellung oder alternative Beschäftigungsmöglichkeiten auf der Straße (Caan et al. 2005, S. 7; Davis 2005a, S. 238). Mit der Zerschlagung der Armee wurde ein wichtiges Symbol der nationalen Einheit zerstört (Jawad 2005, S. 38): „This fact, combined with the perceived humiliation of being forced to live under foreign occupation and, worse still, the prospect of longer-term Shi'i supremacy, led many to take up arms" (Baram 2005, S. 4). Sunnitische Araber, von der neuen Situation enttäuschte Anhänger des alten Systems und überzeugte Islamisten griffen zu den Waffen und bekämpften in der Folge die Besatzungstruppen (Baram 2005, S. 14).

(3) Output-Legitimität durch finanzielle Hilfen: Für Deutschland erwiesen sich die Mittel aus dem European Recovery Program (ERP) als äußerst hilfreich. Die US-Regierung erkannte früh die Notwendigkeit ökonomischer Prosperität für den Wiederaufbau (Benz 1999a, S. 80) sowie für die Stärkung gegenüber „,kommunistischen Verlockungen“ (Graml 1995, S. 30) und entschied noch 1947, dass auch Deutschland Hilfen aus dem ERP erhalten sollte. Die Finanzhilfen des Förderprogramms, im Volksmund kurz „Marshall-Plan" genannt, erzielten einen nicht zu unterschätzenden psychologischen Effekt des Vertrauensgewinns (Thränhardt 1996, S. 61) und unterstützten die wirtschaftliche Öffnung (Lehmann 2000, S. 493). Die Währungsreform von 1948 brachte den Westdeutschen mit der Deutschen Mark zudem eine stabile Währung, die den Warenkonsum rasch ankurbelte und den Grundstein für die soziale Marktwirtschaft legte (Benz 1999a, S. 87). Schließlich waren auch Effekte eines nachholenden Wirtschaftswachstums, des „reconstruction growth“, zu beobachten (Reichel 2002, S. 429). Alle drei Faktoren trugen 
zur Entstehung des „Wirtschaftswunders“ bei. Historiker und Politikwissenschaftler sind sich heute darin einig, dass die Erfolge des wirtschaftlichen Wiederaufbaus erheblich zur raschen Konsolidierung der westdeutschen Demokratie beitrugen und die junge Demokratie legitimierten (Kreikamp 1994, S. 23; Merkel 1999, S. 194-198; Thränhardt 1996, S. 72-78).

Auch im Irak unterstützte die internationale Staatengemeinschaft den Staatsaufbau finanziell. Während der Besatzung stellten allein die USA 20,7 Mrd. US-Dollar Soforthilfe zur Verfügung. Bis 2008 hatten sie insgesamt rund 44,7 Mrd. US-Dollar in den infrastrukturellen Wiederaufbau, die wirtschaftliche Entwicklung und die Demokratisierung investiert (Tarnoff 2008, S. 4). 2,2 Mrd. US-Dollar flossen davon direkt in die ErschlieBung von Erdölfeldern und den Aufbau von Raffinerien. Darüber hinaus hatten schon während der CPA-Besatzung 40 Nationen auf der ersten Geberkonferenz in Madrid im Jahr 2003 rund 15,9 Mrd. US-Dollar zur Verfügung gestellt (Tarnoff 2008, S. 14). Mit den Hilfen verband sich die Erwartung, dass der Irak den Aufbau möglichst rasch aus eigener Kraft würde finanzieren können. Laut UNDG wurden die Aufbaubemühungen allerdings durch die schwierige Sicherheitslage, fehlende Verwaltungskapazitäten vor Ort und den Mangel an Fachkräften, die mit ihren Familien vor Gewalt und Armut geflüchtet waren, erschwert (UNDG 2008, 1). Der UNHCR schätzte, dass sich Ende 2007 rund 2 Mio. Iraker innerhalb des Irak auf der Flucht befanden. Insgesamt rund 2,7 Mio. Iraker waren ins Exil gegangen. Viele waren schon vor 2003 geflüchtet, die Mehrheit jedoch erst nach 2003 (UNHCR 2007, 1-2). Angesichts dieser Zustände lässt sich bisher nicht erkennen, dass die junge irakische Demokratie aus den finanziellen Hilfen eine erhöhte OutputLegitimität generieren konnte.

Abschließend soll noch auf einen Aspekt verwiesen werden, den die US-Planer bei der Vorbereitung ihrer Besatzung des Iraks offenbar nicht einkalkuliert haben und der zusätzlich zu einer Fehleinschätzung des Erfolgs der Demokratisierung in Westdeutschland führte. Die Bilanz des westdeutschen Falles fällt bei genauerem Hinsehen keineswegs so ungetrübt aus, wie die Apologeten eines raschen Demokratisierungserfolges im Irak auf der Basis einer westdeutschen Blaupause suggerierten.

\subsection{Der Preis der externen Demokratisierung}

Der Preis, den Deutschland für die Demokratisierung bezahlte, war hoch. Der OstWest-Konflikt trug erheblich dazu bei, die Redemokratisierung auf westdeutscher Seite $\mathrm{zu}$ forcieren. Briten und US-Amerikaner ließen den Deutschen nach anfänglich strikter Kontrolle viele Freiheiten und versuchten, so wenig wie möglich in die Bildung der Institutionen und den Aufbau der Verwaltung einzugreifen. Ganz anders war die Situation hingegen in der sowjetischen Besatzungszone. Mit aller Härte erzwang die sowjetische Führung in Ostdeutschland ein kommunistisches System. Damit etablierte sie zugleich ein negatives Gegenstück zur Entwicklung der bundesdeutschen Demokratie, was die Demokratisierung auf westdeutscher Seite ihrerseits beschleunigte. Die unterschiedlichen Ansätze zeigten entsprechende Wirkung. Während sich die innen- und wirtschaftspolitische Entwicklung Westdeutschlands an den liberalen Ordnungs- und Freiheitsideen der westlichen Demokratien orientierte, wurde der Osten Deutschlands gezwungenermaßen auf das kommunistische System der Sowjetunion ausgerichtet. In der Folge entschieden 
sich über elf Millionen Ostdeutsche für die Flucht in den Westen, was mit dem Bau der Mauer am 13. August 1961 gestoppt wurde. Die Teilung war damit endgültig vollzogen und die weltpolitische Grenze des „Eisernen Vorhangs“ verlief mitten durch Deutschland. Die rasche Staatsgründung und die Demokratisierung des Westens waren um den hohen Preis der Teilung erkauft worden (Grimm 2010, S. 322).

Zudem behielten sich die Westalliierten ein Kontrollrecht vor. Mit der Gründung der Bundesrepublik im Jahr 1949 trat zugleich das sogenannte „Alliierte Besatzungsstatut“ in Kraft. Es schränkte die Souveränität der jungen Republik erheblich ein und sicherte den alliierten Siegermächten einen „Vorbehalt“ über Deutschland. Das Mitspracherecht bezog sich im Wesentlichen auf die Sicherheit der Besatzungsstreitkräfte und die Rolle der Polizei, auf die Stellung Berlins, auf die Neuordnung der Ländergrenzen, auf die Rückkehr der Flüchtlinge, auf die Entwaffnung und Demilitarisierung, auf die Reparationszahlungen, auf die Zerschlagung der Kartelle, auf die Außenbeziehungen und auf den Außenhandel (Besatzungsstatut 1949). Kein Gesetz konnte ohne die Gegenzeichnung der Hohen Kommissare in Kraft treten (Winkler 2004, S. 138). Dies änderte sich erst mit dem Inkrafttreten der Pariser Verträge nach ihrer Unterzeichnung 1954, die der Bundesrepublik im Deutschlandvertrag ,die volle Macht über ihre inneren und äußeren Angelegenheiten“ zusicherten (Deutschlandvertrag 1954). Ein alliierter Vorbehalt blieb auch nach 1955 bei Fragen bestehen, welche die Wiedervereinigung der beiden deutschen Staaten und die Stadt Berlin betrafen. Die Wiedervereinigung erfolgte erst im Jahr 1990, 41 Jahre nach dem Ende der alliierten Besatzung.

Unklar ist, ob die US-Besatzer 2003 bereit waren, für eine vergleichbar lange Periode amerikanische Soldaten auf irakischem Gebiet zu stationieren, um ihren Beitrag zur Stabilisierung und zum Wiederaufbau des Landes zu leisten. Dies war wohl weder von den Planern beabsichtigt noch von der irakischen Bevölkerung gewünscht. Dass ein hoher Preis an Leben durch die Invasion bezahlt wurde, zeichnete sich hingegen rasch ab. Die Auseinandersetzungen um die Einheit des Staates und die politische Vorherrschaft sowie der Aufstand gegen die Besatzung eskalierten im Irak im Laufe des Jahres 2006 zu einem konfessionsgebundenen Bürgerkrieg (ICG 2006, S. 14-22). Anschläge auf schiitische religiöse Heiligtümer wie etwa auf den Ali al-Hadi- und Al-Hasan al-Askari-Schrein in Samarra im Februar 2006 oder auf die Buratha-Moschee in Bagdad im April 2006 sowie im Gegenzug verübte Racheanschläge auf sunnitische Moscheen in den Folgemonaten verdeutlichen dies (S/2006/137: para. 19; S/2006/945: para. 66). Irakische Sicherheitskräfte gingen massiv gegen sunnitische Aufständische vor, und die multinationalen Truppen setzten ihre militärischen Operationen auf der Suche nach Rebellenmilizen, Al-QaidaTerroristen und Massenvernichtungswaffen mit unverminderter Härte fort (S/2006/360: para. 40). Die Verabschiedung der Verfassung und die Wahl der neuen Regierung hatten die konfessionellen Konfliktlinien eher noch verschärft als abgeschwächt (ICG 2005, S. 5). Die religiös und nationalistisch motivierte Gewalt sowie die Aktivitäten der Sicherheitskräfte und Interventionstruppen hatten verheerende Auswirkungen auf die Zivilbevölkerung. Über die genaue Zahl der getöteten Zivilisten besteht unter Experten, die sich auf verschiedene Erhebungsmethoden stützen, keine Einigkeit. Die Organisation „Iraqi Body Count" (IBC 2008) zählte bis zum 30. Juni 2008 rund 94.000 getötete Zivilisten,ein Expertenteam des MIT kam bis 2006 bereits auf 655.000 getöteter Zivilisten (Burnham et al. 2006, S. 7), und laut einem britischen Team stieg die Zahl bis 2008 auf rund eine 
Million Menschen, die infolge der Intervention, der Aufstände und des Bürgerkriegs ums Leben kamen (Opinion Research Business 2008).

\section{Schlussfolgerungen zum Nutzen historischer Vergleiche}

Im Abschlussbericht der CPA, der im Jahr 2004 kurz vor der überhasteten Übergabe der Amtsgeschäfte an eine irakische Übergangsregierung erschien, versuchten die Verfasser mit einem Vergleich der Fälle Westdeutschland und Irak zu demonstrieren, um wie viel schneller und besser sie im Irak gearbeitet hätten (CPA 2004, S. 71). Auf die Frage eines Journalisten, wie er auf Kritiker reagieren würde, die den USA vorwürfen, sie wendeten nicht genügend Mittel auf, um im Irak den Frieden zu sichern, antwortete der damalige Verteidigungsminister Donald Rumsfeld bei einem National Press Club Luncheon am 10. September 2003, offenbar in Kenntnis dieser Zusammenfassung:

Well, I guess I'd say this: Jerry Bremer, who was here recently, he gave me a piece of paper when I was over in Baghdad this week. He pointed out that the independent central bank of Germany, it took three years after World War II to establish it - it was established in Iraq in two months; that the police in Germany were established after 14 months - in Iraq, they were established in two months; that a new currency in Germany took three years - it took two and a half months in Iraq. The cabinet in Germany took 14 months. Iraq has a cabinet today after four months. Now, how did you go from zero to 55,000 Iraqis providing for their own security? I think the biggest difference is that we now have 24-hour news, and everyone is examining everything every second, and it feels like it's been about four years since the end of the conflict, and it was May 1st. (Laughter.) (Rumsfeld 2003, S. 11).

Rumsfeld hatte sicher Recht mit dem Hinweis auf die signifikanten Unterschiede in der Medienberichterstattung und dem internationalen öffentlichen Interesse an der extern beaufsichtigten Demokratisierung des Jahres 2003 im Vergleich zu derjenigen des Jahres 1945. Allerdings sind seine Einschätzungen über die Funktionstüchtigkeit der ausgewählten irakischen Institutionen mehr als optimistisch. Mit der Distanz von 60 Jahren verblassen zudem die mit der erzwungenen Demokratisierung einhergehenden Probleme. Die besonderen Umstände der westdeutschen Redemokratisierung, die der Ausgang des Zweiten Weltkrieges mit sich brachte, werden leichtfertig übersehen. Drei zentrale Schlussfolgerungen lassen sich aus dem Beispiel des Vergleichs Westdeutschland - Irak ziehen.

Erstens werden historische Vergleiche allzu leicht instrumentalisiert, wenn es politisch opportun erscheint, sich auf vergangene Erfolge zu berufen, um umstrittene Politiken gegenüber anderen Staaten zu rechtfertigen. Dies kann verheerende politische Fehleinschätzungen zur Folge haben, wie das Beispiel der US-Besatzung des Irak zeigt. Von unbesonnenen historischen Analogien, die sich lediglich auf die als positiv eingeschätzten Aspekte eines Einzelfalls stützen, ist dringend abzuraten. Historische Einzelfälle sollten keinesfalls die Grundlage einer riskanten Außenpolitik bilden.

Zweitens kann der methodisch konsequent durchgeführte strukturierte und fokussierte Vergleich (vgl. zur Methode George u. Bennett 2005, S. 67) wichtige Erkenntnisse über 
Erfolgsbedingungen und Hemmnisse extern beaufsichtigter Demokratisierung erbringen. Dies betrifft etwa die strukturellen und kontextuellen Gegebenheiten, unter denen sich die Institutionalisierung und Konsolidierung der Demokratie vollzieht, die Bedeutung eines als mehr oder weniger legitim eingeschätzten externen Eingreifens in die internen Belange eines Gemeinwesens und das fragile Verhältnis zwischen internen und externen Akteuren vor, während und nach der Besatzung.

Die empirische Analyse zeigt, dass die Ausgangssituation für die rasche und dauerhafte Konsolidierung der Demokratie in Westdeutschland nach dem Zweiten Weltkrieg vergleichsweise günstig war: (1) Zwischen den Westalliierten und den westdeutschen politischen Eliten bestand ein substanzieller demokratischer Grundkonsens. Dies war im Irak so nicht gegeben. Ethnische, sozioökonomische und religiöse Konflikte überlagerten dort den Wunsch nach einer raschen Demokratisierung. Zudem wurde eine extern gesteuerte demokratische Konsolidierung des Irak von vielen irakischen Akteuren nicht gewünscht. (2) Die Zahl der relevanten Akteure ist im Vergleich zu Westdeutschland nach dem Zweiten Weltkrieg deutlich gestiegen. Im Irak agieren eine Vielzahl externer Akteure, etwa die Mitglieder der „Koalition der Willigen“, die „Führungsnationen“ in den zentralen Bereichen des Wiederaufbaus, die Vereinten Nationen und ihre Unterorganisationen und unzählige Nichtregierungsorganisationen. Damit können die Lasten des Aufbaus auf mehrere Schultern verteilt werden. Zugleich wird das Angebot internationaler Hilfe aber auch größer, komplexer und weniger transparent. Die Hilfe wird nicht notwendigerweise zielgerichteter vergeben, und der Bedarf an Koordinierung steigt. Je mehr Akteure beteiligt sind, desto größer ist das Risiko von Kooperationsproblemen (vgl. Grimm 2010, S. 70-74). (3) Beide Fälle unterscheiden sich im Legitimitätsprofil der politischen Neuordnung. Deutschland war eindeutig der Verlierer des Zweiten Weltkriegs. Die Politik der Sieger zu akzeptieren war daher notwendig, Alternativen gab es nicht. Angesichts der illegitimen Invasion in den Irak durch die US-geführte „Koalition der Willigen“ wird die externe Demokratisierung im Irak zu Recht in Frage gestellt. In Kenntnis der Kolonialgeschichte und der Abhängigkeit von westlicher Gunst in der Zeit vor dem diktatorischen Regime Saddam Husseins geriet die Besatzung schnell in Verruf, unter dem Deckmantel der Demokratie das externe Oktroi fortzusetzen. Zudem sind (4) die Konfliktlagen im Irak weitaus heterogener als diejenigen in Deutschland 1945. Die ethnische, religiöse und sozioökonomische Fragmentierung ist im Irak deutlich größer, als es in Deutschland trotz der Kriegsschäden und der Flüchtlingsproblematik nach 1945 der Fall war. Im Irak steht, anders als in Deutschland, der Nationalstaat grundsätzlich zur Disposition. Schließlich (5) hat sich die Aufmerksamkeit der internationalen Öffentlichkeit verändert. Der internationale Druck zur Berichtspflicht ist stark gestiegen, und die Arbeit externer Akteure steht unter der permanenten Beobachtung der internationalen Medien, NGOs und Think Tanks. Dies kann ein kritisches und notwendiges Korrektiv sein, macht aber auch Fehler externer Akteure sofort sichtbar und erhöht damit die Unzufriedenheit über die Qualität der externen Präsenz, was sich im Falle des Irak gut beobachten lässt.

Mit dieser Zusammenfassung soll weder gesagt werden, dass die Demokratisierung Westdeutschlands ein zwangsläufiges Ergebnis der Besatzung war, noch, dass die Demokratisierung des Iraks unmöglich ist. Deutlich geworden sein sollte hingegen der dritte wichtige Punkt, der aus der Gegenüberstellung der westdeutschen mit der irakischen Transition unter Besatzung folgt, nämlich: 
Drittens ergeben sich aus vergangenen Erfolgen keine Blaupausen für externe Demokratisierung. Ein allgemeingültiger Demokratisierungspfad unter externer Besatzung existiert nicht, bloß weil sich diese Form der externen Aufsicht in einem vergangenen Fall als erfolgreich erwiesen hat. Ein Vergleich mit einer größeren Anzahl von Fällen der externen Aufsicht über die politische Neuordnung zeigt, dass die damit einhergehende Demokratisierung von Nachkriegsgesellschaften heute grundsätzlich nicht unmöglich, aber doch erheblich aufwändiger und mit einigen Legitimitätsproblemen und Dilemmata behaftet ist (vgl. Grimm 2010). Dabei werden externe Akteure mit der Schwierigkeit leben müssen, dass selbst ein gut organisiertes Projekt zu Staatsaufbau und Demokratisierung, das mit hohem Ressourcenaufwand und einer langfristigen Perspektive betrieben wird, letztlich das unbefriedigende Ergebnisse einer unvollständig konsolidierten Demokratie produzieren kann (vgl. Grimm 2010, S. 336-337). Realistischer ist allerdings, wie auch die Analyse der Besatzung des Irak zeigt, dass ein solches Projekt selten wirklich gut organisiert ist, kaum auf Zustimmung der Bevölkerung trifft, sich schlecht in einem regionalen Kontext verwirklichen lässt, der eine demokratische Staatsform eher gering schätzt, und dass die institutionellen Erblasten nur mit Mühen überwunden werden können. Dies heißt nicht, dass die Demokratisierung des Irak in irakischer Eigenregie unmöglich ist. Aber es heißt, dass die Erfolgschancen einer externen Demokratisierung des Irak auf dem westdeutschen Demokratisierungspfad gering sind.

\section{Primärquellen}

CPA/2003/Order 1. 2003. De-Ba'athification of Iraqi Society, Bagdad, 16. Mai 2003.

CPA/2003/Order 2. 2003. Dissolution of Entities, Bagdad, 23. Mai 2003.

CPA. 2004. An historic review of CPA Accomplishments, http://www.cpa-iraq.org/pressreleases/20040628_historic_review_cpa.doc. Gesehen 30. Juni 2006.

Besatzungsstatut. 1949. Genehmigungsschreiben der drei westlichen Militärgouverneure. In Quellen zur staatlichen Neuordnung Deutschlands 1945-1949, Hrsg. Hans-Dieter Kreikamp, 283284, Darmstadt: Wissenschaftliche Buchgesellschaft.

Deutschlandvertrag. 1954. Vertrag unterzeichnet auf der Konferenz von Paris, 19.-23. Oktober 1954 (Pariser Verträge), in der Fassung vom 23. Oktober 1954. Bundesgesetzblatt II (1955), 306-330.

Frankfurter Dokumente. 1948. Dokumente der drei Militärgouverneure, verlesen und übergeben am 1. Juli 1948 auf einer Konferenz mit den Ministerpräsidenten in Frankfurt. In Quellen zur staatlichen Neuordnung Deutschlands 1945-1949, Hrsg. Hans-Dieter Kreikamp, 243-246, Darmstadt: Wissenschaftliche Buchgesellschaft.

IBC. 2008. Iraqi Body Count Database. http://www.iraqbodycount.org/database/. Gesehen'29. Juli 2008.

S/2006/137. 2006. Report of the Secretary-General Pursuant to Paragraph 30 of Resolution 1546 (2004), 3. März 2006.

S/2006/360. 2006. Report of the Secretary-General Pursuant to Paragraph 30 of Resolution 1546 (2004), 2. Juni 2006.

S/2006/945. 2006. Report of the Secretary-General Pursuant to Paragraph 30 of Resolution 1546 (2004), 5. Dezember 2006.

S/RES/661. 1990. UN Security Council Resolution, 6. August 1990 (Irak).

S/RES/688. 1991. UN Security Council Resolution, 5. April 1991 (Irak). 
SC/7664. 2003. Press Release SC/7664, Security Council, 4707th Meeting. Iraq Cooperating with Disarmament Procedures, but Many Banned Weapons Remain Unaccounted for, Inspectors Tell Security Council. „Immediate, Unconditional and Active“ Cooperation Needed to Resolve Questions; France, China, Russian Federation, Germany Support Continued Inspection Process, 14. Februar 2003.

SC/7696. 2003. Press Release SC/7696, Security Council, 4721th Meeting. Importance of Humanitarian Aid for Iraq Stressed, as Security Council Members Voice Different Views on Disarmament Process. Council Hears Report of Chief UN Weapons Inspector, 19. März 2003 (Irak).

UNDHA. 1995. UN Consolidated Inter-Agency Humanitarian Cooperation Programme for Iraq: Mid-term Review, 21. September 1995.

The Yalta Conference. 1945. Protocol of Proceedings of Crimea Conference, 11. Februar 1945.

\section{Sekundärquellen}

Abelshauser, Werner. 1983. Wirtschaftsgeschichte der Bundesrepublik Deutschland (1945-1980). Frankfurt: Suhrkamp.

Abelshauser, Werner. 1999. Kriegswirtschaft und Wirtschaftswunder. Deutschlands wirtschaftliche Mobilisierung für den Zweiten Weltkrieg und die Folgen für die Nachkriegszeit. Vierteljahreshefte für Zeitgeschichte 47 (4): 503-538.

Allawi, Ali A. 2007. The occupation of Iraq. Winning the war, losing the peace. New Haven: Yale University Press.

Baram, Amatzia. 1997. Neo-tribalism in Iraq. Saddam Hussein's tribal policies 1991-1996. International Journal of Middle East Studies 29 (1): 1-31.

Baram, Amatzia. 2005. Who are the Insurgents? Sunni Arab rebels in Iraq. Special Report 134, April 2005. Washington: United States Institute of Peace Press.

Benjamin, Daniel. 2003. Condi's Phony History. Sorry, Dr. Rice, Postwar Germany was Nothing like Iraq http://www.slate.com/id/2087768/. Zugegriffen: 29. Aug. 2003.

Benz, Wolfgang. 1999a. Die Gründung der Bundesrepublik. Von der Bizone zum souveränen Staat. München: dtv.

Benz, Wolfgang. Hrsg. 1999b. Deutschland unter alliierter Besatzung 1945-1949/1955. Berlin: Akademie Verlag.

Blanchard, Christopher M., Kenneth Katzman, Carol Migdalovitz, Alfred Prados, und Jeremy Sharp. 2008. Iraq. Regional perspectives and U.S. Policy, CRS Report for Congress, updated 4 April 2008. Washington: Congressional Research Service.

Bracher, Karl Dietrich. 1955. Die Auflösung der Weimarer Republik. Eine Studie zum Problem des Machtverfalls in der Demokratie. Villingen: Ring Verlag.

Bungenstab, Karl-Ernst. 1970. Umerziehung zur Demokratie? Re-education-Politik im Bildungswesen der US-Zone 1945-1949. Düsseldorf: Bertelsmann Universitätsverlag.

Burnham, Gilbert, Shannon Doocy, Elizabeth Dzeng, Riyadh Lafta, und Les Roberts. 2006. The human cost of the war in Iraq. A mortality study 2002-2006. Cambridge, Mass.: MIT.

Bush, George W. 2002. The president's state of the union address, the United States capitol, Washington, DC, 29 January 2002. http://www.whitehouse.gov/news/releases/2002/01/20020129$11 \mathrm{html}$. Zugegriffen: 21. Juli 2008.

Byman, Daniel. 2004. Insecuring Iraq. The national interest, Sommer 2004:15-18.

Caan, Christina, Beth C. DeGrasse, Paul Hughes, und Daniel Serwer. 2005. Is this any way to run an occupation? An investigation of the development of governance in post-conflict Iraq. Paper Presented at the Conference „Interim Governments: Institutional Bridges to Peace and Democracy?“, 14.-15. Juli 2005. Monterey, CA: National Security Affairs Department and Naval Postgraduate School. 
Cederman, Lars-Erik, Simon Hug, und Andreas Wenger. 2008. Democratization and war in political science. Democratization 15 (3): 509-524.

Cockayne, James, und David M. Malone. 2006. Creeping unilateralism. How operation provide comfort and the no-fly zones of 1991 and 1992 paved to way for the Iraq crisis 2003. Security Dialogue 37 (1): 123-141.

Colvin, Ian. 1947. Exporting democracy to Germany. The National Review 129 (Juli/Dezember): 109-117.

Crocker, Bathsheba, Rebecca Linder, und Christopher Zirpoli. 2004. Progress or peril? Measuring Iraq's reconstruction. Public opinion in Iraq, November 2004. Post-conflict reconstruction project. Washington: The Post-Conflict Reconstruction Project.

Croissant, Aurel. 2002. Von der Transition zur defekten Demokratie. Wiesbaden: Westdeutscher Verlag.

Davis, Eric. 2005a. History matters: Past as prologue in building democracy in Iraq. Orbis 49 (2): 229-244.

Davis, Eric. 2005b. Memories of state. Politics, history, and collective identity in modern Iraq. Berkeley: University of California Press.

Dawisha, Adeed. 2004. Iraq: Setbacks, advances, prospects. Journal of Democracy 15 (1): 5-20.

Denison, Andrew B. 2003. Unilateral oder multilateral? Motive der amerikanischen Irakpolitik. Aus Politik und Zeitgeschichte. (B 24-25/2003): 17-24.

Diamond, Larry. 2005. Squandered victory. The American occupation and the bungled effort to bring democracy to Iraq. New York: Times Books.

Dobbins, James F., John G. McGinn, Keith Crane, Seth G. Jones, Rollie Lal, Andrew Rathmell, Rachel Swanger, und Anga Timilsina. 2003. America's role in nation-building. From Germany to Iraq. Washington: Rand Corporation.

Dodge, Toby. 2003. Inventing Iraq. The failure of nation-building and a history denied. New York: Columbia University Press.

Dorn, Walter L. 1958. Die Debatte über die amerikanische Besatzungspolitik für Deutschland (1944-1945). Vierteljahreshefte für Zeitgeschichte 6 (1): 60-77.

Duelfer, Charles. 2004. Comprehensive report of the special advisor to the DCI on Iraq's WMD, 30. September 2004. Washington.

Dunn, David H. 2003. Myths, motivations and ,misunderestimations'. The Bush administration and Iraq. International Affairs 79 (2): 279-297.

Ebsworth, Raymond. 1960. Restoring democracy in Germany. The British Contribution. London: Stevens \& Sons Limited.

Elster, Jon. 2004. Closing the books. Transitional justice in historical perspective. Cambridge: Cambridge University Press.

Eschenburg, Theodor. 1974. Der bürokratische Rückhalt. In Die Zweite Republik. 25 Jahre Bundesrepublik Deutschland - eine Bilanz, Hrsg. Richard Löwenthal und Hans-Peter Schwarz, 64-94. Stuttgart: Busse-Seewald.

Fait, Barbara. 2001. Kontrollierte Demokratisierung. In Amerikanische Besatzung und deutsche Politik. In Die USA und Deutschland im Zeitalter des Kalten Krieges. Band 1: 1945-1968/ Band 2: 1968-1990. Ein Handbuch, Hrsg. Detlef Junker, 100-111. Stuttgart: Deutsche Verlagsanstalt.

Farouk-Sluglett, Marion, und Peter Sluglett. 2003. Iraq since 1958. From revolution to dictatorship. London: Tauris.

Feldman, Noah. 2004. What we owe Iraq. War and the ethics of nation building. Princeton: Princeton University Press.

Fontan, Victoria. 2006. Polarization between occupier and occupied in Post-Saddam Iraq. Colonial humiliation and the formation of political violence. Terrorism and Political Violence 18 (2): 217-238. 
Frei, Norbert. 2002. Adenauer's Germany and the Nazi past. New York: Columbia University Press.

Fürstenau, Justus. 1969. Entnazifizierung. Ein Kapitel deutscher Nachkriegspolitik. Neuwied: Luchterhand.

George, Alexander L., und Andrew Bennet. 2005. Case studies and theory development in the social sciences. Cambridge: MIT Press.

Gimbel, John. 1968. Amerikanische Besatzungspolitik in Deutschland 1945-1949. Frankfurt: S. Fischer.

Graml, Hermann. 1995. Die Besatzungspolitik der Alliierten in Deutschland 1945-1949. Aus Politik und Zeitgeschichte. (B 25-33/1995): 25-33.

Grimm, Sonja. 2010. Erzwungene Demokratie. Politische Neuordnung nach militärischer Intervention unter externer Aufsicht. Baden-Baden: Nomos.

Guelke, Adrian. 2003. Negotiations and peace processes. In Contemporary Peacemaking. Conflict, violence and peace processes, Hrsg. John Darby und Roger Mac Ginty, 53-64. Houndmills: Palgrave.

Hartzell, Caroline D., Matthew Hoddie, und Donald Rothchild. 2001. Stabilizing the peace after civil war. An investigation of some key variables. International Organization 55 (1): 183-208.

Herz, John H. 1982. Denazification and related policies. In From dictatorship to democracy. In Coping with the legacies of authoritarianism and totalitarianism, Hrsg. John H. Herz, 15-38. Westport-London: Greenwood Press.

Higley, John, und Michael L. Burton. 1989. The elite variable in democratic transitions and breakdowns. American Sociological Review 54 (1): 17-32.

Hoffmann-Lange, Ursula. 1998. Germany: Twenthieth-century turning points. In Elites, crises, and the origins of regimes, Hrsg. Mattei Dogan und John Higley, 169-188. Lanham: Rowman \& Littlefield.

Hoffmann, Stefan-Ludwig. 2007. Zwischen Krieg und Frieden. Über demokratische Besatzung. Merkur. Deutsche Zeitschrift für europäisches Denken 61 (10): 973-977.

Huntington, Samuel. 1991. The third wave. Democratization in the late twentieth century. Norman: University of Oklahoma Press.

ICG. 2003. Iraq's constitutional challenge, Middle East Report $N^{\circ} 19$, 13. November 2003. Brüssel: International Crisis Group.

ICG. 2005. Unmaking Iraq: A constitutional process gone awry, Middle East Briefing No. 19, 26. September 2005. Brüssel: International Crisis Group.

ICG. 2006. The next Iraqi War? Sectarianism and civil conflict, Middle East Report N52, 27. Februar 2006. Brüssel: International Crisis Group.

Jackson, Robert H. 2004. International engagement in war-torn countries. Global Governance 10 (1): $21-36$.

Jawad, Pamela. 2005. Der Irak nach dem Dritten Golfkrieg. In Nach dem Dritten Golfkrieg: Sicherheitspolitische Analysen zu Verlauf und Folgen des Konflikts. Wissenschaft \& Sicherheit, Band 1, Hrsg. Björn Kilian, Christian Tobergte, und Simon Wunder, 37-46. Berlin: Berliner Wissenschafts-Verlag.

Kagan, Robert, und William Kristol. 2004. Democracy now. The weekly standard. 17. Mai 2004.

Kirchner, Henner. 2003. Neubeginn oder „neue Katastrophe“? Auswirkungen des Irakkrieges auf die arabischen Nachbarstaaten. Aus Politik und Zeitgeschichte. (B 24-25/2003): 56-62.

Kramer, Heinz. 2007. Die Interessen der Türkei. In Regionale (Neu-)Ordnung im Nahen und Mittleren Osten und die Rolle externer Akteure, SWP-Studie 7, Hrsg. Muriel Asseburg. Berlin: Stiftung Wissenschaft und Politik.

Kreikamp, Hans-Dieter. 1994. Quellen zur staatlichen Neuordnung Deutschlands 1945-1949. Darmstadt: Wissenschaftliche Buchgesellschaft.

Krieger, Leonard. 1968. The potential for democratization in occupied Germany: A problem in historical projection. Public Policy 17:27-58. 
Krieger, Leonard. 1979. Das Interregnum in Deutschland: März-August 1945. In Die Bundesrepublik Deutschland, Hrsg. Wolf-Dieter Narr und Dietrich Thränhardt, 26-46. Königstein: Verlagsgruppe Athenäum.

Lehmann, Axel. 2000. Der Marshall-Plan und das neue Deutschland. Die Folgen amerikanischer Besatzungspolitik in den Westzonen. Münster: Waxmann.

Lepsius, M. Rainer. 1978. From fragmented party democracy to government by emergency decree and national socialist takeover: Germany. In The breakdown of democratic regime, Vol. 2, Hrsg. Juan J. Linz und Alfred Stephan, 34-79. Baltimore: The Johns Hopkins University Press.

Levitsky, Steven, und Lucan A. Way 2006. Linkage versus leverage. rethinking the international dimension of regime change. Comparative Politics 38 (4): 379-400.

Lipset, Seymour M. 1959. Some social requisites of democracy. Economic development and political legitimacy. American Political Science Review 53 (1): 69-105.

Lipset, Seymour M. 1962. Soziologie der Demokratie. Neuwied: Hermann Luchterhand Verlag.

Mack, Andrew and Asif Khan. 2000. The efficacy of UN sanctions. Security Dialogue 31 (3): 279-292.

Makiya, Kanan. 1998. Republic of fear: The politics of modern Iraq. Berkeley: University of California Press.

Mann, Michael. 2003. Das rasche Scheitern des Imperium Americum. Internationale Politik 9 (10): $52-56$.

Marr, Phebe. 2004. The modern history of Iraq. Boulder: Westview Press.

Massarrat, Mohssen. 2005. Demokratisierung des Greater Middle East. Aus Politik und Zeitgeschichte. (B 45/2005): 30-37.

Merkel, Wolfgang. 1999. Systemtransformation. Opladen: Leske \& Budrich.

Merkel, Wolfgang, Hans-Jürgen Puhle, Aurel Croissant, Claudia Eicher, und Peter Thiery. 2003. Defekte Demokratie. Band 1: Theorie, Opladen: Leske \& Budrich.

Merkl, Peter. 1968. Allied strategies of effecting political change and their reception in occupied Germany. Public Policy 17 (1): 59-103.

Montgomery, John D. 1957. Forced to be Free. The artificial revolution in Germany and Japan. Chicago: The University of Chicago Press.

Müller, Rolf-Dieter. 2004. Der Zweite Weltkrieg. Stuttgart: Klett-Cotta.

Opinion Research Business. 2008. New analysis ,confirms' 1 Million + Iraq casualties, press release, 28. Januar $2008 \mathrm{http} / / / \mathrm{www} . o p i n i o n . c o . u k / N e w s r o o m \_d e t a i l s . a s p x ? N e w s I d=88$. Zugegriffen: 29. Juli 2008.

Papagianni, Katia 2007. State building and transitional politics in Iraq. The perils of a top-down transition. International Studies Perspectives 8 (3): 253-271.

Piel, Edgar. 1996. Spuren der NS-Ideologie im Nachkriegsdeutschland. In Freundliche Feinde? Die Alliierten und die Demokratiegründung in Deutschland, Hrsg. Heinrich Oberreuter und Jürgen Weber, 145-167. München: Günter Olzog Verlag.

Przeworski, Adam. 1991. Democracy and the market. Political and economic reforms in Eastern Europe and Latin America. Cambridge: Cambridge University Press.

Przeworski, Adam, und Henry Teune. 1970. The logic of comparative social inquiry. New York: Wiley-Interscience.

Przeworski, Adam, und Fernando Limongi. 1997. Modernization. Theories and facts. World Politics 49 (1): 155-183.

Prowe, Diethelm. 1993. German democratization as conservative restabilization. The impact of american policy. In American policy and the reconstruction of West Germany, Hrsg. Jeffry M. Diefendorf, Axel Frohn, und Hermann-Josef Rupieper, 307-329. Cambridge: Cambridge University Press.

Putnam, Robert D. 1993. Making democracy work. Civic traditions in modern Italy. Princeton: Princeton University Press. 
Reichel, Richard 2002. Germany's postwar growth. Economic miracle or reconstruction boom? The Cato Journal 21 (3): 427-442.

Rice, Condoleezza. 2003. Namensartikel der Beraterin des amerikanischen Präsidenten für Nationale Sicherheit, Condolezza Rice, veröffentlicht am 7. August 2003 in der Washington Post. Internationale Politik 9 (10): 111-113.

Rotberg, Robert I. 2004. The failure and collapse of nation-states. Breakdown, prevention, and repair. In When States Fail. Causes and Consequences, Hrsg. Robert I. Rotberg, 1-49. Princeton: Princeton University Press.

Rowat, Colin. 2001. How sanctions hurt Iraq. Middle East Report Online http://www merip.org/ mero/mero080201 html. Zugegriffen: 6. Juli 2001.

Rumsfeld, Donald. 2003. Remarks at national press club luncheon, 10. September $2003 \mathrm{http} / / /$ www.defenselink.mil/cgi-bin/dlprint.cgi?http://www.defenselink mil/transcripts/2003/ tr20030910secdef0661 html. Zugegriffen: 20. Juni 2006.

Schaller, Christian. 2006. Peacebuilding und „, ius post bellum“. Völkerrechtliche Rahmenbedingungen der Friedenskonsolidierung nach militärischen Interventionen, SWP Studie 2006/S11. Berlin: Stiftung Wissenschaft und Politik.

Schoch, Bruno. 2006. Demokratisierung von außen - ein Ding der Unmöglichkeit? Zwischen langfristigen Prozessen und gewaltsamen Anstößen. Kommune. Forum für Politik, Ökonomie, Kultur 24 (3): 16-27.

Schreiber, Gerhard 2007. Der Zweite Weltkrieg. München: Beck.

Shirer, William L. 1981. The rise and fall of the third reich. A history of Nazi Germany. New York: Touchstone.

SIGIR. 2006. Special inspector general for Iraq reconstruction. Iraq reconstruction. Lessons in contracting and procurement, Juli 2006. Washington/Baghdad: SIGIR.

Sponeck, Hans-Christof von. 2005. Ein anderer Krieg. Das Sanktionsregime der UNO im Irak. Hamburg: Hamburger Edition.

Stansfield, Gareth. 2007. Iraq. People, history, politics. Cambridge: Polity Press.

Tarnoff, Curt. 2008. Iraq. Reconstruction assistance. CRS Report for Congress, updated 22 May 2008. Washington: Congressional Research Service.

Thränhardt, Dietrich. 1996. Geschichte der Bundesrepublik Deutschland. Frankfurt a.M.: Suhrkamp.

Tomuschat, Christian. 2003. Völkerrecht ist kein Zweiklassenrecht. Der Irak-Krieg und seine Folgen. Vereinte Nationen 51 (2): 41-46.

Tripp, Charles. 2005. A History of Iraq. Cambridge: Cambridge University Press.

Ullmann, Hans-Peter. 1995. Das Deutsche Kaiserreich 1871-1918. Frankfurt a.M.: Suhrkamp.

Vanhanen, Tatu. 1983. A study on the emergence of democracy. European Journal of Political Research 11 (2): 223-227.

Wallich, Herny C. 1955. Triebkräfte des deutschen Wiederaufstiegs. Frankfurt a.M.: Fritz Knapp Verlag.

Wallich, Herny C. 1979. Das „Deutsche Wunder“- eine Übersicht. In Die Bundesrepublik Deutschland. Entstehung, Entwicklung, Struktur, Hrsg. Wolf-Dieter Narr und Dietrich Thränhardt, 60-74. Königstein: Verlagsgruppe Athenäum.

Walter. Barbara. 1997. The critical barrier to civil war settlement. International Organization 51 (3): 335-365.

Wehler, Hans-Ulrich. 2003. Deutsche Gesellschaftsgeschichte. Vierter Band. Vom Beginn des Ersten Weltkrieges bis zur Gründung der beiden deutschen Staaten 1914-1949. München: Verlag C. H. Beck.

Whitehead, Laurence. 2004. Democratization with the benefit of hindsight. The changing international components. In The UN role in promoting democracy. Between ideals and reality, Hrsg. Edward Newman und Roland Rich, 135-165. Tokyo: United Nations University Press. 
Winkler, Heinrich August. 2004. Der lange Weg nach Westen II. Deutsche Geschichte 1933-1990. Bonn: Bundeszentrale für politische Bildung.

Wolfowitz, Paul. 2004. Prepared statement for the house armed services committee. As prepared for delivery for the house armed services committee by deputy secretary of defense Paul Wolfowitz, Washington, DC, 22. Juni 2004 http://www.defenselink.mil/cgi-bin/dlprint.cgi?http:// www.defenselink mil/speeches/2004/sp20040622-depsecdef0521 html. Zugegriffen: 20. Juni 2006.

Woods, Kevin M. 2006. Iraqi perspectives project. A view of operation Iraqi freedom from Saddam's senior leadership. Norfolk: Joint Center for Operational Analysis.

Zapf, Wolfgang. 1966. Wandlungen der deutschen Elite. Ein Zirkulationsmodell deutscher Führungsgruppen 1919-1961. München: Piper.

Zuhur, Sherifa D. 2006. Iran, Iraq, and the United States: The new triangle's impact on sectarianism and the nuclear threat. Carlisle, Pennsylvania: Strategic Studies Institute. 